\title{
RESOLUBILIDADE DA COMUNICAÇÃO FARMACÊUTICO-PACIENTE NA PRÁTICA DA DISPENSAÇÃO DE MEDICAMENTOS
}

\author{
Tassila Brito Amorim $^{1}$; Tatiane de Oliveira Silva Alencar ${ }^{2}$ \\ 1. Bolsista PROBIC, Graduando em Farmácia, Universidade Estadual de Feira de Santana, e-mail: \\ tassilamorim@hotmail.com \\ 2. Orientador, Departamento de Saúde, Universidade Estadual de Feira de Santana, e-mail: tatifarmauefs@ yahoo.com,br
}

PALAVRAS-CHAVE: assistência farmacêutica; dispensação; comunicação

\section{INTRODUÇÃO}

A dispensação de medicamentos consiste no ato farmacêutico de distribuir um ou mais medicamentos em resposta a uma prescrição, constituindo uma oportunidade de $\mathrm{o}$ farmacêutico contribuir para o uso racional de medicamentos (URM), pois a partir da interação por meio da comunicação com o usuário, é possível identificar a necessidade e fornecer as orientações sobre o medicamento prescrito e educação em saúde (GALATO et al., 2008; COSTA, et al., 2014).

Desse modo, considerando as ações da Assistência Farmacêutica nos municípios brasileiros em suas atividades assistenciais, na perspectiva da prática farmacêutica focada principalmente ao usuário na etapa da dispensação, o estudo apresenta como questionamento: Como ocorre o processo de comunicação farmacêutico-usuário no ato da dispensação de medicamentos no SUS de Feira de Santana-BA? Diante desse questionamento, foram delimitados como objetivo geral do estudo: discutir a resolubilidade da comunicação farmacêutico-usuário de medicamentos nas farmácias do SUS em Feira de Santana-Bahia; e como objetivos específicos: discutir o processo de dispensação de medicamentos realizado por farmacêuticos nas farmácias públicas em Feira de Santana-BA e compreender como se dá a comunicação entre o farmacêutico e o usuário no processo de dispensação.

\section{METODOLOGIA}

O estudo tem caráter exploratório e qualitativo, foi realizado nas farmácias públicas em Feira de Santana, que tinham farmacêuticos. A. Os participantes do estudo foram os farmacêuticos dos serviços de saúde que dispensam medicamentos ( 1 farmacêutico do Programa de Controle da Hanseníase; 1 farmacêutico do Programa de Controle das DST/AIDS -1 farmacêutico do Programa de Parkinson). As técnicas de coleta de dados foram a entrevista semiestruturada, por meio de roteiro e a observação sistemática (da farmácia e do processo de dispensação), por meio de roteiro de observação. O tratamento dos dados foi feito utilizando o método de análise de conteúdo. A pesquisa obedeceu à Resolução no 466 de 12 de dezembro de 2012, do Ministério da Saúde. Sendo assim, aqueles que concordaram em participar da pesquisa assinaram o Termo de Consentimento Livre e Esclarecido.

\section{RESULTADOS E/OU DISCUSSÃO}

Os resultados apresentados envolveram primeiramente a dispensação de medicamentos, e, em seguida a comunicação nesta prática farmacêutica.

\section{Dispensação de medicamentos}

A dispensação de medicamentos, enfatizada nas políticas farmacêuticas, é o ato profissional farmacêutico de proporcionar o medicamento a um usuário, geralmente como resposta a uma receita elaborada por um profissional autorizado e nesse ato, o farmacêutico informa e orienta o mesmo sobre o uso adequado do medicamento. Esse processo tem o intuito de entregar o medicamento correto, na dosagem e quantidade prescrita, com instruções para o uso correto, 
assegurando a qualidade do produto. Este momento é fundamental para o Uso Racional de Medicamentos (URM) e, por isso é uma etapa na qual a comunicação é relevante e os profissionais de saúde envolvidos com esta atividade devem estar capacitados para fornecer todas essas informações de maneira adequada e acessível à linguagem do usuário (BRASIL, 2001). Na prática, o farmacêutico é o profissional responsável pela direção do processo diante do seu vínculo histórico e indissociável do medicamento e por ser o detentor do conhecimento acerca dessa tecnologia de saúde. Assim considera-se que a relação, orientação e acompanhamento do usuário do medicamento seja a etapa mais relevante do trabalho farmacêutico. Entretanto, essa atividade é ainda incipiente nos serviços públicos de saúde, pois há dificuldades deste profissional em dispor seu conhecimento para ações junto à população, inviabilizando a avaliação de seu trabalho na qualidade dos serviços e melhoria da saúde dos usuários (ARAÚJO, et al., 2008). No campo em estudo, especificamente nas farmácias dos programas de parkinson, hanseníase, DST/Aids. Esses programas fornecem assistência à saúde aos pacientes, incluindo a médica e a farmacêutica. Constatou-se por meio das entrevistas e observação que para alguns farmacêuticos a dispensação consiste apenas na entrega do medicamento. Para outros, a orientação medicamentosa faz parte deste processo, porém é realizada consideravelmente na primeira dispensação, após diagnóstico confirmado pelo médico, sendo que nas dispensações subseqüentes a orientação não é fornecida, devido aos usuários dos medicamentos não desejarem por estarem expostos no serviço. Também evidenciou-se a preocupação dos farmacêuticos com as atividades administrativas que envolvem o processo de dispensação.

Sobre as condições estruturais do serviço para a realização da dispensação, (materiais de apoio e estrutura física), os farmacêuticos afirmaram que o espaço físico não é apropriado para o atendimento ao paciente e que não utilizavam materiais de apoio na dispensação.

\section{Comunicação}

Conforme Rossignoli, Godoy e Correr (2013), a comunicação na área da saúde implica o estudo e o uso de estratégias e técnicas de comunicação para informar, orientar e influenciar os indivíduos com o objetivo da promoção, proteção e recuperação da saúde visando a um serviço de saúde resolutivo. Assim, as habilidades de comunicação do profissional permitem que o usuário assistido receba um cuidado científico e humanizado com informações sobre sua doença e seu tratamento, possibilitando ao usuário a participação ativa no processo terapêutico. No âmbito do cuidado em saúde, a interação farmacêutico-usuário é determinante para a qualidade do serviço e para resolubilidade das demandas de saúde (LYRA; MESQUITA, 2012). Rossignoli, Godoy, Correr (2013), apresentam como eventos relevantes na comunicação farmacêutica o acolhimento, o aconselhamento, a empatia, a escuta ativa e a assertividade.

No cenário analisado observou-se que os elementos (acolhimento, aconselhamento, empatia, escuta ativa e assertividade) que envolvem a comunicação farmacêutica não são efetivos na prática. As condições estruturais do serviço público não promovem, por exemplo, o acolhimento, que compreende principalmente a linguagem não verbal, no qual o usuário precisa se sentir em um ambiente especializado de atendimento à saúde, sendo que, o farmacêutico deve expressar-se interessado e comprometido com as necessidades do usuário. Pelo contrário, no campo de estudo observou-se que não havia um local reservado e especializado para o atendimento ao usuário do serviço, não havia, portanto, o acolhimento. As farmácias nos serviços estudados dividiam o espaço com atividades administrativas. Percebeu-se também uma comunicação deficiente, pois os farmacêuticos demonstraram se preocupar com a documentação necessária para a dispensação. 
Além da orientação verbal, também é importante a orientação escrita para a educação em saúde, para o uso adequado do medicamento e para a resolubilidade da comunicação farmacêutico-usuário, entretanto, em relação ao fornecimento de orientação escrita, evidenciou-se que os farmacêuticos não forneciam.

A empatia, a escuta ativa e a assertividade, elementos constantes na boa comunicação, não foram possíveis de serem interpretadas na comunicação farmacêutico-usuário visto que não foi possível entrevistar usuários de medicamentos dos serviços estudados, devido a esses pacientes apresentarem patologias complexas, que envolvem questões sociais como preconceito e discriminação, portanto estes aspectos da comunicação não foram possíveis de serem analisados.

Em relação às dificuldades na dispensação de medicamentos apresentadas pelos farmacêuticos identificou-se, como já foi expresso, a limitação da estrutura física, ou seja, um local adequado para tal prática, pela fala do entrevistado E1 e E3, respectivamente: "O espaço físico não é apropriado para o atendimento ao paciente" e "Na minha realidade, a quantidade de pacientes já é grande, então às vezes eu não tenho espaço físico pra ta acondicionando corretamente as medicações, os preservativos e a gente tem que dá um jeitinho. Então a gente pede um pouco menos, porque não vai ter onde colocar. A sala querendo ou não é um pouco pequena".

Identificou-se a acomodação e passividade do profissional diante de um serviço inconsistente, pela fala do entrevistado E1: "A gente não tem muita dificuldade porque a gente já está acostumado". A falta de medicamentos também foi citada como dificuldade pela fala do entrevistado E1: Agora mesmo está faltando medicamentos do Programa desde janeiro. Desde janeiro que não tem disponibilidade no estoque".

Para o entrevistado E2, uma dificuldade é a barreira do usuário, que muitas vezes não quer a orientação, como demonstrado na fala a seguir:

"O paciente em uso de talidomida, o que eu tenho observado ao longo desses anos no serviço, é um paciente que não quer esperar, ele não quer se expor, ele tem alterações na pele, tem pacientes cronificado, com muitas lesões na pele, é um paciente que não se expõe, não quer conversa, malmente ele quer assinar o que ele tem que assinar"

Estudos mostram que as habilidades comunicativas do farmacêutico produzem resultados positivos em saúde, especialmente relacionados à farmacoterapia, sobretudo nos estudos com pacientes diabéticos e hipertensos, no qual o acompanhamento farmacoterapêutico realizado por meio da relação farmacêutico-usuário é eficaz (ROSSIGNOLI; GODOY; CORRER, 2013).

Entretanto, o trabalho do farmacêutico nas farmácias do Sistema Único de Saúde está restrito à gestão do medicamento, ou seja, tem o objetivo de selecionar, programar e garantir o acesso ao medicamento, e quando existe dispensação com orientação adequada, nota-se que são instruções relacionadas à informação quantitativa sobre o medicamento e é fornecida por meio de um diálogo unilateral, no qual apenas o farmacêutico dita o que acha importante (ARAÚJO; FREITAS, 2005).

Todavia as possibilidades visualizadas na dispensação vão além da entrega de medicamentos e do fornecimento de orientações técnicas. Esta é a oportunidade de se estabelecer vínculos, de acolher o usuário e a sua família, de aplicar as tecnologias leves a partir da comunicação efetiva, da construção e manutenção de uma relação terapêutica 
resolutiva e produtora do cuidado em saúde (GALATO, et al., 2008; ALENCAR; NASCIMENTO; ALENCAR, 2011).

\section{CONSIDERAÇÕES FINAIS}

O estudo proporcionou a discussão do processo de dispensação, porém não foi possível compreender como se dá o processo de comunicação entre o farmacêutico e o usuário no processo de dispensação no campo do estudo, devido às limitações da estrutura do serviço e também postura do profissional. Sugere-se a mudança de postura do farmacêutico a partir da utilização dos elementos da comunicação em seu exercício profissional.

\section{REFERÊNCIAS}

ALENCAR, T. O. S.; NASCIMENTO, M. A. A. do; ALENCAR, B. R. Assistência

Farmacêutica no SUS: articulando sujeito, saberes e práticas. Feira de Santana: UEFS Editora, 2011.

ARAÚJO, A. L. A.; UETA, J. M.; FREITAS, O. Assistência farmacêutica como um modelo tecnológico em atenção primária à saúde. Rev. Ciênc. Farm. Básica Apl., v. 26, n.2, p. 8792, 2005.

ARAÚJO, et al., Perfil da assistência farmacêutica na atenção primária do Sistema Único de Saúde. Ciênc. \& Saúde Coletiva, p. 611-617, 2008.

BRASIL, Política Nacional de Medicamentos. Ministério da Saúde, 2001

GALATO, D. et. al. A dispensação de medicamentos: uma reflexão sobre o processo para prevenção, identificação e resolução de problemas relacionados à farmacoterapia. Rev. Bras. de Ciênc. Farma., São Paulo, v. 44, n. 3, jul./set. 2008.

LYRA, D. P. J.; MESQUITA, A. R. As bases da dispensação racional de medicamentos para farmacêuticos. São Paulo: Pharmabooks, 2012.

OLIVEIRA, L. C. F.; ASSIS, M. M. A.; BARBONI, A. R. Assistência Farmacêutica no Sistema Único de Saúde: da Política Nacional de Medicamentos à Atenção Básica à Saúde.

Ciêc. \& Saúde Coletiva, Rio de Janeiro, v. 15, n. 1, p. 3561-3567, nov. 2010.

OLIVEIRA, L. C. F.; ASSIS, M. M. A.; NASCIMENTO, M. A. A. Assistência

Farmacêutica na Atenção Básica: os desafios da gestão para o acesso integral. Feira de Santana: UEFS Editora, 2014.

POSSAMAI, F. P.; DACOREGGIO, M. S. Habilidade de comunicação com o paciente no processo de atenção farmacêutica. Trab. Educ. Saúde, Rio de Janeiro, v. 5, n. 3, nov. 2008.

ROSSIGNOLI; GODOY; CORRER. A prática farmacêutica na farmácia comunitária. Porto Alegre: Artmed, 2013. 